\title{
Lower Baseline Performance but Greater Plasticity of Working Memory for Carriers of the Val Allele of the COMT Val ${ }^{158}$ Met Polymorphism
}

\author{
Martin Bellander and Lars Bäckman \\ Karolinska Institutet, Stockholm, Sweden and \\ Stockholm University
}

\author{
Brit-Maren M. Schjeide and Lars Bertram \\ Max Planck Institute for Molecular Genetics, Berlin, Germany \\ Ulman Lindenberger \\ Max Planck Institute for Human Development, Berlin, Germany
}

\author{
Tian Liu \\ Max Planck Institute for Human Development, Berlin, Germany
}

Florian Schmiedek

German Institute for International Educational Research (DIPF), Frankfurt am Main, Germany

Martin Lövdén

Karolinska Institutet, Stockholm, Sweden, Stockholm University, and Max Planck Institute for Human Development, Berlin, Germany

\begin{abstract}
Objective: Little is known about genetic contributions to individual differences in cognitive plasticity. Given that the neurotransmitter dopamine is critical for cognition and associated with cognitive plasticity, we investigated the effects of 3 polymorphisms of dopamine-related genes (LMX1A, DRD2, COMT) on baseline performance and plasticity of working memory (WM), perceptual speed, and reasoning. Method: One hundred one younger and 103 older adults underwent approximately 100 days of cognitive training, and extensive testing before and after training. We analyzed the baseline and posttest data using latent change score models. Results: For working memory, carriers of the val allele of the COMT polymorphism had lower baseline performance and larger performance gains from training than carriers of the met allele. There was no significant effect of the other genes or on other cognitive domains. Conclusions: We relate this result to available evidence indicating that met carriers perform better than val carriers in WM tasks taxing maintenance, whereas val carriers perform better at updating tasks. We suggest that val carriers may show larger training gains because updating operations carry greater potential for plasticity than maintenance operations.
\end{abstract}

Keywords: cognitive plasticity, COMT, dopamine, latent change score models, working memory

Individual variation in cognitive function is, in part, genetically determined. Estimates of genetic influences are about $50 \%$ for working memory (WM; Ando, Ono, \& Wright, 2001; Wright et al., 2001, although, see Friedman et al., 2008, where genes accounted for almost all variance), between $30 \%$ and $60 \%$ for episodic memory (EM; Papassotiropoulos \& De Quervain, 2011), 30\% to

This article was published Online First May 12, 2014.

Martin Bellander and Lars Bäckman, Aging Research Center, Karolinska Institutet, Stockholm, Sweden and Stockholm University; Tian Liu, Center for Lifespan Psychology, Max Planck Institute for Human Development, Berlin, Germany; Brit-Maren M. Schjeide and Lars Bertram, Max Planck Institute for Molecular Genetics, Berlin, Germany; Florian Schmiedek, Center for Research on Education and Human Development, German Institute for International Educational Research (DIPF), Frankfurt am Main, Germany; Ulman Lindenberger, Center for Lifespan Psychology, Max Planck Institute for Human Development; Martin Lövdén, Aging Research Center, Karolinska Institutet and Stockholm University, and Center for Lifespan Psychology, Max Planck Institute for Human Development.

Correspondence concerning this article should be addressed to Martin Bellander, Aging Research Center, Karolinska Institutet, Gävlegatan 16, SE-113 30, Stockholm, Sweden. E-mail: martin.bellander@ki.se
$80 \%$ for intelligence (Deary, Johnson, \& Houlihan, 2009), and $40 \%$ to $80 \%$ for perceptual speed (PS; Posthuma, Mulder, Boomsma, \& De Geus, 2002).

The neurotransmitter dopamine (DA) plays a major role in cognitive functioning. This is evident from investigations on patient populations, experimental work with animals, pharmacological challenges, and molecular imaging research (Bäckman, Lindenberger, Li, \& Nyberg, 2010; Bäckman, Nyberg, Lindenberger, Li, \& Farde, 2006; Cropley, Fujita, Innis, \& Nathan, 2006). Given the relation between DA and cognition, and the high heritability of cognitive functions, many studies on candidate genes and cognition have focused on DA-related polymorphisms (Savitz, Solms, \& Ramesar, 2006).

Besides being related to cognition in general, DA has also been implicated in the ability to improve cognitive function from training, especially in relation to WM (Bäckman \& Nyberg, 2013). The causes of individual differences in effects of cognitive training on performance are relatively unexplored, as studies have mainly focused on group differences (e.g., patients vs. healthy controls; training groups vs. control groups). Theoretical work has highlighted the need to investigate the genetics associated with individuals' potential for plastic change (Belsky et al., 2009; Lövdén, 
Bäckman, Lindenberger, Schaefer, \& Schmiedek, 2010; Mercado, 2008). Two recent studies investigated the effects of polymorphisms in DA-related genes on training-related gains in WM. These studies demonstrated that carrying advantageous alleles for the DA transporter gene (associated with DA reuptake in striatum; Brehmer et al., 2009) and the LMX1A gene (related to the number of DA-producing neurons in midbrain; Bellander et al., 2011) were associated with larger gains from five weeks of WM training. These patterns were observed in the presence of equivalent pretraining WM performance for the different allelic variants.

Here, we examine genetic effects on individual differences in baseline cognitive abilities and the potential to improve on these abilities in response to training. Three single nucleotide polymorphisms (SNPs) in DA-related genes were selected: rs4657412 in the LMX1A gene, rs6277 in the DRD2 gene, and rs4680 in the COMT gene ( $\left.\mathrm{Val}^{158} \mathrm{Met}\right)$. The reason for choosing the SNPs in the COMT and DRD2 was the large previous literature on their effects on cognition or cognitive plasticity in combination with the fact that their functional effect is partly known. The SNP in the LMX1A gene were chosen to try to replicate an earlier finding of an association with gains from cognitive training, from a study with a small sample (Bellander et al., 2011).

LMX1A is a transcription factor that is pivotal for the correct differentiation of mesencephalic DA neurons during the embryonic stage (Friling et al., 2009). The rs4657412 SNP for LMX1A has been linked not only to training-related gains in WM (Bellander et al., 2011), but also to risk of Parkinson's disease (Bergman et al., 2009).

The rs6277 SNP for the DRD2 gene affects D2 receptor availability in both striatal (Hirvonen et al., 2004, 2005) and extrastriatal (Hirvonen et al., 2009) regions. In a PET study, Bäckman and colleagues (2011) found that binding potential to the D2 receptor decreased after WM training, indicating larger release of endogenous DA. Evidence suggests that individuals carrying the DRD2 allele associated with more D2 receptors perform better in various cognitive tasks than those who carry the allele associated with lower receptor density (Bolton et al., 2010; Li et al., 2013; Papenberg et al., 2013).

In frontal cortices, DA is mainly catabolized by the catecholO-methyl transferase (COMT) enzyme. The Val ${ }^{158}$ Met polymorphism in the COMT gene results in a valine to methionine substitution with activity increasing in a dose-response fashion depending on the number of val alleles. For val homozygotes, the enzyme shows a threefold increase in activity compared with met homozygotes (Tunbridge, Harrison, \& Weinberger, 2006). A meta-analysis found an association between the met allele and better performance in the Wisconsin Card Sorting Task in healthy subjects (Barnett, Jones, Robbins, \& Müller, 2007), whereas another meta-analysis indicated an association between the met allele and higher IQ, but failed to show any relation to WM (Barnett, Scoriels, \& Munafò, 2008). Importantly, met carriers seem to perform better at tasks requiring maintenance of information in WM, whereas val carriers perform better in updating tasks (Colzato, Waszak, Nieuwenhuis, Posthuma, \& Hommel, 2010; Krugel, Biele, Mohr, Li, \& Heekeren, 2009). These differences can be accounted for by the distinction between tonic and phasic components of DA signaling. Phasic DA is important to updating whereas tonic DA is important to maintenance. The balance between tonic and phasic DA is shifted toward a stronger phasic component in val carriers (Bilder, Volavka, Lachman, \& Grace, 2004). In many cognitive tasks, these differences may cancel each other out, the net effect being equivalent performance in val and met alleles. Importantly, in a recent study by Colzato and colleagues (2013), val homozygotes showed greater transfer to task-switching (i.e., flexibility) than met carriers from videogame training.

Using a sample of 204 younger and older adults from the COGITO study who underwent cognitive training for approximately 100 days, we investigated the effect of the three DA SNPs on WM, PS, and reasoning. Both cognitive baseline performance and degree of improvement from training were investigated for trained tasks and for untrained tasks assessing transfer of learning. The potential interaction between age group and genes was also of interest, as genetic effects have been suggested to be magnified in aging (Lindenberger et al., 2008). In studies of genetic effects on cognition, target cognitive abilities are often assessed with modest degrees of validity and reliability. To overcome this problem, we adopted a structural equation modeling approach, using a latent change score model (LCSM; McArdle \& Nesselroade, 1994). This statistical technique separates measurement error and task-specific variance from individual differences in ability, thereby reducing problems with raw difference scores, such as low measurement reliability and regression toward the mean effects. It also allows for testing whether the cognitive construct measured by the tasks is invariant across measurement occasions.

\section{Method}

\section{Participants}

In the COGITO study, 219 participants were recruited. Of these, 15 dropped out, leaving 204 participants in the final sample: 101 younger adults (aged 20-31 years) and 103 older adults (aged 65-80 years; see Table 1 for descriptive information of the sample). Participants received between 1450 and 1950 EUR for participation. The payment was based on the number of completed training sessions and the number of days it took to complete the required number of sessions. For a detailed description of the design, the sample, and the tasks, see Schmiedek, Lövdén, and Lindenberger (2010).

\section{Procedure}

Participants underwent approximately 101 days of cognitive training (younger adults: $M=100.8, S D=2.6$, range $=87-109$; older adults: $M=101.0, S D=2.7$, range $=90-106)$. Each day participants trained for about one hour on 12 computerized tasks:

Table 1

Subject Characteristics by Age Group

\begin{tabular}{lcc}
\hline \multicolumn{1}{c}{ Characteristic } & Younger adults & Older adults \\
\hline Females & $51.5 \%$ & $49.5 \%$ \\
Age & $25.6(2.7)$ & $71.3(4.1)$ \\
Years of education & $16.1(3.2)$ & $13.6(3.6)$ \\
WAIS Digit-Symbol & $60.3(9.5)$ & $43.6(9.0)$ \\
Accuracy Spot-a-Word Test & $0.66(0.10)$ & $0.81(0.10)$ \\
\hline
\end{tabular}

Note. $\quad$ WAIS $=$ Wechsler Adult Intelligence Scale. 
three WM tasks, three EM tasks, and six PS tasks. A battery assessing the trained tasks as well as untrained tasks assessing WM near and far transfer, EM transfer, PS transfer, and reasoning was administered before and after the 100 days of training.

\section{Trained Tasks}

The trained WM tasks consisted of alpha span, numerical memory updating, and spatial $n$-back. The trained EM tasks included word recall, number-noun recall, and recall of spatial position of objects. The six PS tasks involved three two-choice reaction time (RT) tasks with different materials; odd/even numbers, consonant/ vowel letters, and symmetric/asymmetric combinations of lines, and three comparison tasks where participants either had to compare two strings of digits, two strings of consonants, or two three-dimensional figures. For all training tasks, except the three PS comparison tasks, difficulty levels were individualized by adjusting the presentation times based on the participant's performance at baseline testing. The difficulty level was then maintained throughout the training (for details, see Schmiedek et al., 2010).

\section{Transfer Tasks}

The transfer tasks were either computerized or taken from the paper-and-pencil Berlin Intelligence Structure Test (BIS; Jäger, Süß, \& Beauducel, 1997). The near-transfer WM tasks were similar to the trained WM tasks but differed in materials. Three WM complex span tasks were used to assess far transfer. For EM transfer, nine tasks were taken from the BIS (three for each content domain) and one was a word paired-associate learning task, resulting in four indicators. The three indicators for PS transfer were constructed using nine tasks from the BIS (three per content domain). Four indicators were used for reasoning using Raven's Advanced Progressive Matrices and nine tasks from the BIS, again three tasks for each content domain (for details, see Schmiedek et al., 2010).

\section{Genotyping}

Blood was drawn for genotyping from those participants consenting at a follow-up session, after the main study was completed, resulting in a subsample of 125 participants being genotyped (47 younger adults, 78 older adults). DNA was extracted from peripheral blood using standard methods. This study refers to genotype data from three common SNPs in LMX1A (rs4657412, intronic), DRD2 (rs6277, synonymous [Pro219Pro]), and COMT (rs4680, nonsynoymous [Val158Met]). All SNPs were genotyped at Max Planck Institute for Molecular Genetics on $5 \mathrm{ng}$ of DNA per subject in 384-well microtiter plates using commercially available real time polymerase chain reaction (RT-PCR) allelic discrimination assays based on TaqMan chemistry (specifically assay-IDs C__1503267_20 [rs4657412], C_11339240_10 [rs6277], and C_25746809_50 [rs4680]) using custom-made OpenArray multiplex genotyping arrays (Applied Biosystems, Forster City, CA) following the manufacturer's recommendations as described in Schjeide et al. (2011). When compared with control DNA samples with genotyping data available from the International HapMap Project (of which 32 were here included on each 384-well plate), the average error rate of the genotyping experiments was $<1 \%$.
Genotypes for all three polymorphisms were in agreement with Hardy Weinberg Equilibrium ( $p$ s $>.05$ ). For the LMX1A SNP, 123 participants were successfully genotyped, with the following distribution: 71 A/A, $43 \mathrm{~A} / \mathrm{G}, 9 \mathrm{G} / \mathrm{G}$. For the DRD2 SNP, 124 participants: $69 \mathrm{~A} / \mathrm{A}, 42 \mathrm{~A} / \mathrm{C}, 13 \mathrm{C} / \mathrm{C}$. For the COMT SNP, 124 participants: $41 \mathrm{met} / \mathrm{met}, 56 \mathrm{met} / \mathrm{val}, 27 \mathrm{val} / \mathrm{val}$.

\section{Data Analysis}

Using raw difference scores when studying change over time is problematic because the reliability of change scores tends to be low, reflecting the influence of measurement error. One solution to this problem is to model change latently using SEM, as latent variables are free of measurement error. Latent factors represent the common variance among the observed variables (tasks) and are constructed by regressing the observed variables onto the latent factor. In the LCSM, a latent factor measuring the construct is specified for each of two time points, T1 and T2. To obtain the latent change in the construct between $\mathrm{T} 1$ and $\mathrm{T} 2$, a latent difference variable (Diff) is constructed by constraining both the regression path from T1 to T2 and Diff to T2 to 1 . In this manner, T2 is defined as the sum of $\mathrm{T} 1$ and Diff, and consequently Diff represents the change from $\mathrm{T} 1$ to $\mathrm{T} 2$. The T1 and Diff variables can then be regressed on the predictors (in this case the selected SNPs) to see if the predictors have an effect on initial level and change in the construct, respectively. For a schematic representation of the model, see Figure 1.

The LCSMs were estimated using Mplus, version 7 (Muthén \& Muthén, 1998-2012). Factors for each domain were created using the tasks belonging to that domain as indicators. Though only a subsample of the participants was genotyped, the full sample was

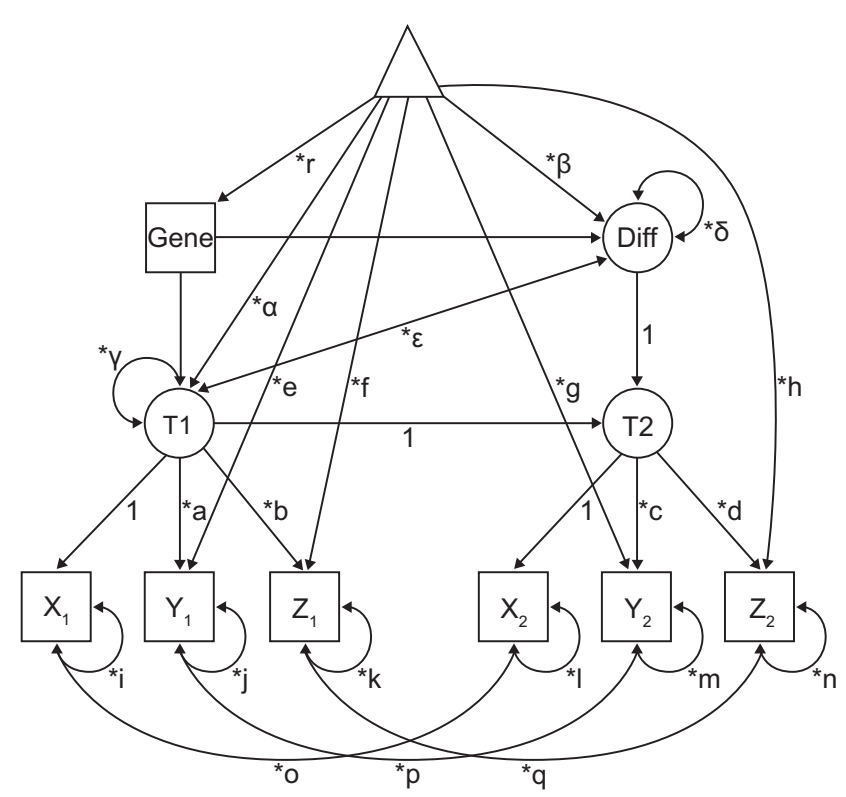

Figure 1. Schematic representation of the latent change score model (LCSM) used in the analyses. Boxes represent observed variables, and circles represent latent variables. The triangle represents estimated means. Single-headed arrows represent regressions, and double-headed arrows represent variances and covariances. 
used in the LCSMs. By including participants who were not genotyped, the larger sample size increased the stability in the estimation of the cognitive structure. The genetic data from the genotyped subsample were then projected into the LCSMs as predictors of the latent variables. The genotype sample did not differ from the full sample on any of the tasks used in the models, neither at baseline nor at posttest (Cohen's $d$ : -0.10 to 0.18 ; $p$ s $>$ $0.19)$.

The interpretability of SEM model comparisons is greatly enhanced if the relations between measurements and latent factors are invariant over time, between groups, or both (Meredith \& Teresi, 2006). Measurement invariance (MI) makes it more likely but does not guarantee that the same construct is measured. Testing the degree of MI in SEM consists in a series of nested models comparisons, allowing for likelihood ratio tests of statistical significance. The higher the level of invariance, the higher the likelihood that the same construct is measured over time or between groups. Weak factorial invariance means that the factor loadings are invariant. This is tested by restricting the loadings to be the same over time or between groups, and comparing the $\chi^{2}$ of this model with the model where they are free to vary. If the model fit is significantly different between the restricted and the free model, weak factorial invariance cannot be assumed. In Figure 1, weak invariance over time would be tested by comparing the fit of the unrestricted model to the model making the following restrictions: $a=c, b=d$. Strong invariance means that not only factor loadings but also the intercepts are invariant, (restrictions: $a=c, b=d, e=$ $g, f=h$ ). Finally, strict invariance means that in addition to the requirements of strong invariance, the residual variances of the indicator are also invariant (restrictions: $a=c, b=d, e=g, f=$ $h, i=l, j=m, k=n)$.

Here, our strategy was to use the highest level of invariance admissible for each model. If no level of MI could be shown to hold, the model was not used in further analysis. MI was tested through the $\chi^{2}$-difference between the restricted and the free model. MI was first tested between age groups using a multiple group model; if between-Group MI could not be rejected, the groups were collapsed and MI over time was tested.

When testing MI between age groups, the PS transfer was the only model where strict MI could not be rejected $\left(\Delta \chi_{24}^{2}=56.4\right.$, $p=.058)$. Also, strong MI was rejected for all other models. Factorial MI was a tenable assumption for EM training $\left(\Delta \chi_{14}^{2}=\right.$ $14.0, p=.13)$, WM near transfer $\left(\Delta \chi_{14}^{2}=14.7, p=.71\right)$, WM far transfer $\left(\Delta \chi_{14}^{2}=22.3, p=.15\right)$, and reasoning $\left(\Delta \chi_{36}^{2}=35.2, p=\right.$ $.18)$. The models for which all levels of MI was rejected were dropped from further analysis. These were WM training, PS training, and EM transfer.

Across time points strict MI could be assumed for WM near transfer $\left(\Delta \chi_{12}^{2}=11.7, p=.68\right)$, PS transfer $\left(\Delta \chi_{12}^{2}=29.7, p=\right.$ $.15)$, and reasoning $\left(\Delta \chi_{25}^{2}=29.2, p=.35\right)$. Strong MI was rejected for the other models. Factorial MI was not rejected for WM far transfer $\left(\Delta \chi_{7}^{2}=14.5, p=.053\right)$. No level of MI could be assumed for EM training, which was therefore dropped from further analysis.

To summarize, the models used in later analyses of genetic effects were WM near transfer, WM far transfer, PS transfer, and reasoning. For these models, the three polymorphisms were entered individually as predictors of baseline performance and change across the 100 days of practice. The genetic data were entered into the model as a predictor of latent baseline performance and change and coded as the number of minor alleles $(0,1$, or 2). When appropriate, latent effect sizes were calculated by dividing the mean of the latent difference factor for each genotype group by the pooled latent standard deviation at pretest for the groups.

The fit of the models were evaluated using the Root Mean Square Error of Approximation (RMSEA). For this fit index, a cut-off value of 0.06 (lower value indicating better fit) has been proposed (Hu \& Bentler, 1999). The RMSEA was below this cut-off for most of the models. It was slightly higher than recommended for four of the 12 models (the three PS models and the WM far transfer model with the COMT SNP). Therefore, the results from these models should be interpreted with caution (see Table 2 for RMSEA for all models). The significance testing for each parameter was done by examining the $\chi^{2}$-difference between the model with the parameter restricted to zero and the model where it was freely estimated. Testing the two parameters (effect of the SNP on baseline performance and change) for four constructs with three SNPs resulted in a total of $24 \chi^{2}$-tests. The alpha level was corrected for multiple comparisons using Bonferroni correction, resulting in an alpha level of 0.002 .

\section{Results}

The general approach used was to model change for all latent cognitive variables and examine how each gene predicted baseline performance and change. No significant effects were found for LMX1A or DRD2 in any of the models. For COMT, one significant effect was obtained after correcting for multiple comparisons, namely the effect on the change score parameter for WM near transfer $\left(\Delta \chi_{1}^{2}=14.19, p=.00017\right)$. Here, each val allele increased the gain between baseline and posttest (see Figure 2A). The effect sizes of change was 0.20 for the met/met, 0.39 for the val $/ \mathrm{met}$, and 0.67 for the val/val genotype group. With respect to baseline performance, no significant COMT effect was obtained when controlling for multiple comparisons, although the effect was below the conventional .05 level $\left(\Delta \chi_{1}^{2}=4.84, p=.028\right)$. Here, the met allele was predictive of higher baseline performance (see Figure $2 \mathrm{~B}$ ). The effect size for the difference in baseline performance compared to the met/met genotype group was -0.11 for $\mathrm{val} / \mathrm{met}$, and -0.66 for val/val. There were no effects of COMT on any of the other measures. The model for WM near transfer and

Table 2

RMSEA for the LCSMs for Each Cognitive Ability and SNP

\begin{tabular}{|c|c|c|c|c|c|c|}
\hline \multirow[b]{2}{*}{ RMSEA } & \multicolumn{3}{|c|}{ WM near transfer } & \multicolumn{3}{|c|}{ WM far transfer } \\
\hline & LMX1A & COMT & DRD2 & LMX1A & COMT & DRD2 \\
\hline \multirow[t]{2}{*}{ RMSEA } & 0.000 & 0.030 & 0.038 & 0.059 & 0.064 & 0.059 \\
\hline & \multicolumn{3}{|c|}{ PS transfer } & \multicolumn{3}{|c|}{ Reasoning transfer } \\
\hline RMSEA & 0.071 & 0.086 & 0.070 & 0.021 & 0.037 & 0.000 \\
\hline
\end{tabular}

Note. RMSEA $=$ root mean square error of approximation; LCSM $=$ latent change score model; SNP = single nucleotide polymorphism; $\mathrm{WM}=$ working memory. LMXIA, COMT, and DRD2 are dopaminerelated genes. 

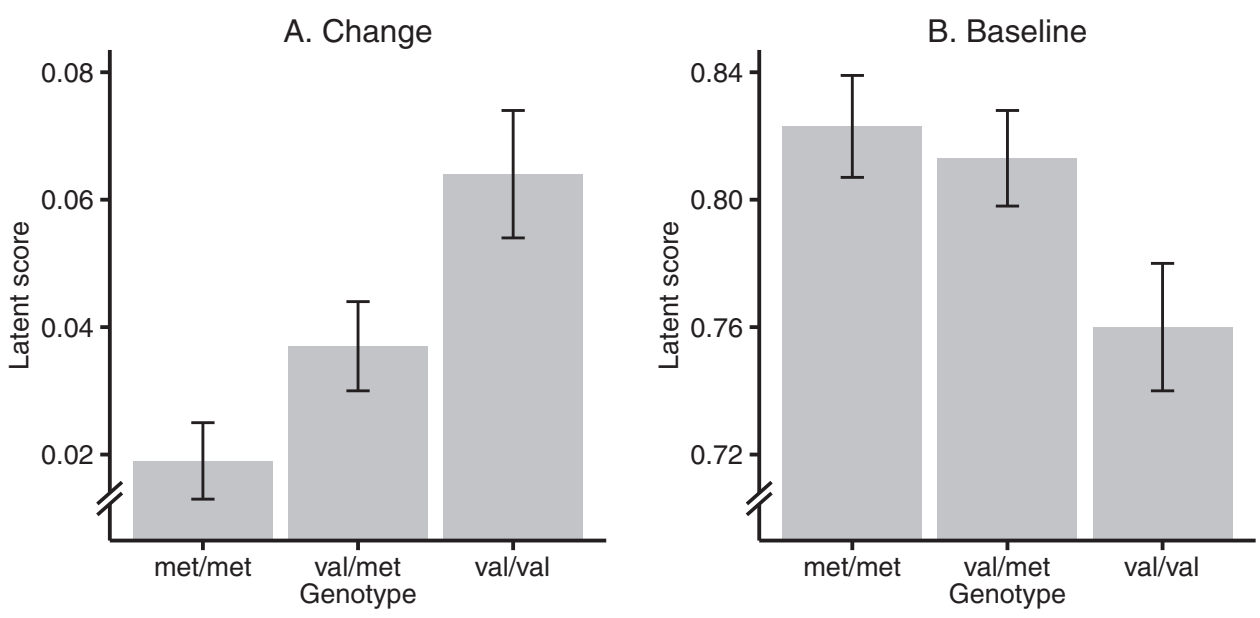

Figure 2. (A) Latent WM near transfer scores for change between baseline and post training, across the three genotype groups. Error bars represent standard errors. (B) Latent WM near transfer scores at baseline across the three genotype groups. Error bars represent standard errors.

COMT had good fit, RMSEA $=0.030$. The $p$ values from all analyses are presented in Figure 3. We proceeded by examining the COMT SNP in more detail.

The effect of COMT on WM near transfer was further investigated by including age group in the model. There was a main effect of age group on both latent factors (baseline: $\Delta \chi_{1}^{2}=166.51, p<$ .001 ; change: $\Delta \chi_{1}^{2}=5.26, p=.022$ ), indicating that old adults showed lower performance at baseline and gained less from training than young adults. Adding age group into the model did not alter the main effects of COMT (baseline: $\Delta \chi_{1}^{2}=4.90, p=.027$; change: $\left.\Delta \chi_{1}^{2}=14.03, p=.00018\right)$. The COMT $\times$ Age Group interaction was then entered into the model to investigate whether the effect of COMT was modulated by age. The interaction was not significant (baseline: $\Delta \chi_{1}^{2}=0.00, p=1.00$; change: $\Delta \chi_{1}^{2}=$ $0.05, p=.83$ ), indicating that the effect of COMT on change did not differ reliably as a function of age group.

The trained WM tasks did not allow for modeling in latent space because of the lack of construct invariance between measurement occasions and across age groups. To ascertain that the WM findings were not restricted to the near transfer construct, we pro-

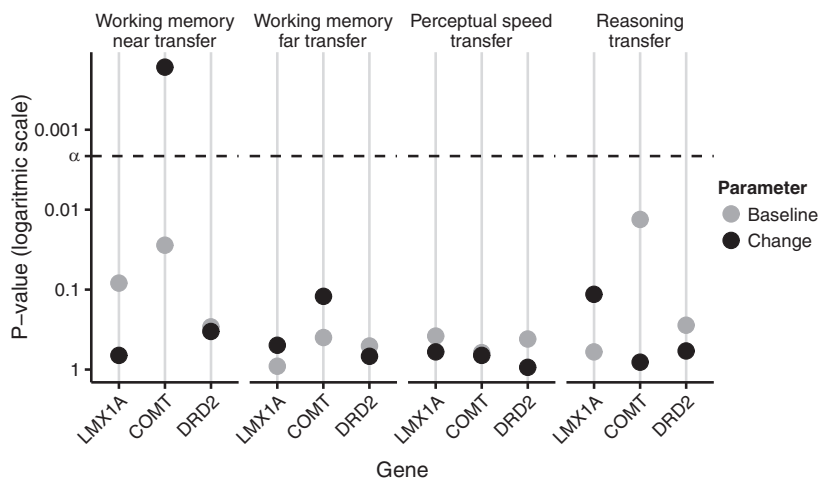

Figure 3. $p$ values (logarithmic scale) for the baseline and change parameters in the LCSMs for each cognitive ability and SNP. ceeded by investigating the trained WM tasks at the observed level. To be able to analyze the results using ANOVAs, the genotypes were dichotomized as val/val versus any met, following earlier work on training-related gains (Stroth et al., 2010). The effect of genotype, age, and time were tested using $2 \times 2 \times 2$ mixed ANOVAs for each of the three trained WM tasks. Baseline differences between genotypes were tested using independent sample $t$ tests. For all three tasks, there were significant effects of age group and time (all $p s<0.001$ ). For Alpha span, genotype groups differed at baseline, $t(48.13)=3.50, p=.001$. There was also an effect of gene, $F(1,120)=4.80, p=.030, \eta^{2}=0.04$, and an Age Group $\times$ Time interaction, $F(1,120)=5.48, p=.021, \eta^{2}=0.04$. The Time $\times$ Gene interaction was just above conventional significance, $F(1,120)=3.90, p=.051, \eta^{2}=0.03$. Genotypes differed at baseline also in the updating task, $t(55.55)=2.64, p=.011$. In this task, there was an Age Group $\times$ Gene interaction, $F(1,120)=$ $8.20, p=.005, \eta^{2}=0.06$, as well as a Time $\times$ Gene interaction, $F(1,120)=7.63, p=.007, \eta^{2}=0.06$. Although only a trend for baseline differences, $t(52.52)=1.72, p=.092$, the pattern of results was similar for the $n$-back, with Age Group $\times$ Gene, $F(1$, $120)=6.24, p=.014, \eta^{2}=0.05$, and Time $\times$ Gene interactions, $F(1,120)=4.18, p=.043, \eta^{2}=0.03$. In general, the results from these analyses were in line with those from the LCSM of WM near transfer, in that val homozygotes started lower but gained more from baseline to post training compared to met carriers.

\section{Discussion}

We investigated the effects of three DA-related genetic polymorphisms (LMX1A, DRD2, and COMT) on baseline cognitive performance as well as gains across 100 days of practice using LCSMs. No significant effects were obtained in the analyses of DRD2 or LMX1A. However, the COMT gene had an effect on baseline performance as well as on plasticity in WM near transfer: The val allele was predictive of lower baseline performance, but larger gains in response to training. A similar pattern was seen at the manifest level for the trained WM tasks that did not allow for 
latent modeling. These patterns did not differ reliably between young and old adults.

The most common pattern in studies investigating cognitive effects of the COMT gene is that met carriers perform better than val carriers (Barnett et al., 2007). This is in line with the present results, where met carriers showed higher WM performance at baseline. However, there are findings deviating from this pattern, likely reflecting the fact that the different COMT alleles have both advantages and disadvantages (Barnett et al., 2008). Val carriers have been shown to outperform met carriers in updating tasks, whereas this pattern is reversed for tasks requiring maintenance of information in WM (Bilder et al., 2004; Colzato et al., 2010; Krugel et al., 2009; Nolan, Bilder, Lachman, \& Volavka, 2004). There is also evidence that videogame training induced transfer to task-switching is greater for val homozygotes than met carriers (Colzato et al., 2013). The results from the present study fit well with these results. These characteristics of val and met carriers are related to the distinction between tonic and phasic components of DA signaling (Grace, 1991). Tonic DA reflects constant lowfrequency firing of DA neurons resulting in more stable representations, whereas phasic DA involves high-frequency burst firing, thought to be more important for updating of WM representations (Bilder et al., 2004). In val carriers, the relative levels of tonic and phasic DA are shifted toward a stronger phasic component (Bilder et al., 2004), and val carriers have higher synthesis of DA in midbrain (Tunbridge et al., 2006).

There are indications that the updating component is generally more trainable than the maintenance component (Jolles, Grol, Van Buchem, Rombouts, \& Crone, 2010). Also, the procedure of individualizing presentation times of the WM tasks (to levels as fast as possible while keeping performance above chance) did put particular emphasis on the efficiency of updating operations. Thus, the greater WM plasticity of val carriers reported here could be accounted for by these individuals having an advantage in the updating component of WM, which is more plastic than the maintenance component and particularly tapped by the training regime. On this view, participants' improvements may have been driven by increases in updating efficiency leading to stronger reliance on updating after training compared with baseline. This notion fits well with findings that updating training results in increased blood flow (Dahlin, Stigsdotter Neely, Larsson, Bäckman, \& Nyberg, 2008) and increased release of DA in the striatal complex (Bäckman et al., 2011). It is also consistent with computational work based on animal studies that phasic DA is important to long-term plastic changes in the brain (Sheynikhovich, Otani, \& Arleo, 2011). This line of reasoning could also explain why no differences were found in the other cognitive domains for the COMT genotypes, as these are not dependent on the mechanisms of maintenance and updating to the same extent as WM. The specific effect on WM, indicating discriminant validity, is compatible with the fact that COMT mainly affects DA functioning in frontal cortices and the frontostriatal loop. These are crucial for WM functioning, whereas PS relies more heavily on other brain areas.

No effects were found for either DRD2 or LMX1A, although these genes have been reported to influence cognitive performance (DRD2; Bolton et al., 2010; Li et al., 2013; Papenberg et al., 2013) and plasticity (LMX1A; Bellander et al., 2011) in earlier work. One concern in the present study is the large number of statistical tests performed. Although correcting for multiple comparisons, this procedure greatly increases the risk of type II errors. It might well be that the DRD2 and LMX1A genes do indeed affect cognitive performance and plasticity, but that the effects are too small to be detected given the restricted sample size in relation to the number of statistical tests performed.

Another point of concern is that for several of the LCSMs, only weak factorial MI could be assumed. Although strict MI could be assumed across time for the WM near transfer model, across age groups only weak factorial MI could be assumed. This should be held in mind when interpreting the results, as this could potentially mean that the constructs were measured differently in the two age groups.

To gain further insight into the differential effects of the COMT genotype on WM and WM plasticity, further research would first need to confirm the present results of differential allelic effects on baseline performance and gains. An interesting follow-up on the present results would be to train participants in a task in which the maintenance and updating components could be clearly separated. This would allow investigating whether the two WM components are differently affected by training in the COMT genotype groups. Based on our interpretation of the present findings, we predict that val homozygotes gain more in the updating component whereas met homozygotes might show a larger gain in the maintenance component.

\section{References}

Ando, J., Ono, Y., \& Wright, M. (2001). Genetic structure of spatial and verbal working memory. Behavior Genetics, 31, 615-624. doi:10.1023/ A:1013353613591

Bäckman, L., Lindenberger, U., Li, S., \& Nyberg, L. (2010). Linking cognitive aging to alterations in dopamine neurotransmitter functioning: Recent data and future avenues. Neuroscience and Biobehavioral Reviews, 34, 670-677. doi:10.1016/j.neubiorev.2009.12.008

Bäckman, L., \& Nyberg, L. (2013). Dopamine and training-related working-memory improvement. Neuroscience \& Biobehavioral Reviews, Advance online publication. doi:10.1016/j.neubiorev.2013.01.014

Bäckman, L., Nyberg, L., Lindenberger, U., Li, S.-C., \& Farde, L. (2006). The correlative triad among aging, dopamine, and cognition: Current status and future prospects. Neuroscience and Biobehavioral Reviews, 30, 791-807. doi:10.1016/j.neubiorev.2006.06.005

Bäckman, L., Nyberg, L., Soveri, A., Johansson, J., Andersson, M., Dahlin, E., . . Rinne, J. O. (2011). Effects of working-memory training on striatal dopamine release. Science, 333, 718. doi:10.1126/science .1204978

Barnett, J. H., Jones, P. B., Robbins, T. W., \& Müller, U. (2007). Effects of the catechol-O-methyltransferase Val158Met polymorphism on executive function: A meta-analysis of the Wisconsin Card Sort Test in schizophrenia and healthy controls. Molecular Psychiatry, 12, 502-509. doi:10.1038/sj.mp.4001973

Barnett, J. H., Scoriels, L., \& Munafò, M. R. (2008). Meta-analysis of the cognitive effects of the catechol-O-methyltransferase gene Val158/ 108Met polymorphism. Biological Psychiatry, 64, 137-144. doi: 10.1016/j.biopsych.2008.01.005

Bellander, M., Brehmer, Y., Westerberg, H., Karlsson, S., Fürth, D., Bergman, O., . . Bäckman, L. (2011). Preliminary evidence that allelic variation in the LMX1A gene influences training-related working memory improvement. Neuropsychologia, 49, 1938-1942. doi:10.1016/j .neuropsychologia.2011.03.021

Belsky, J., Jonassaint, C., Pluess, M., Stanton, M., Brummett, B., \& Williams, R. (2009). Vulnerability genes or plasticity genes? Molecular Psychiatry, 14, 746-754. doi:10.1038/mp.2009.44 
Bergman, O., Håkansson, A., Westberg, L., Belin, A. C., Sydow, O., Olson, L., . . N Nissbrandt, H. (2009). Do polymorphisms in transcription factors LMX1A and LMX1B influence the risk for Parkinson's disease? Journal of Neural Transmission, 116, 333-338. doi:10.1007/s00702009-0187-z

Bilder, R. M., Volavka, J., Lachman, H. M., \& Grace, A. A. (2004). The catechol-O-methyltransferase polymorphism: Relations to the tonicphasic dopamine hypothesis and neuropsychiatric phenotypes. Neuropsychopharmacology, 29, 1943-1961. doi:10.1038/sj.npp.1300542

Bolton, J. L., Marioni, R. E., Deary, I. J., Harris, S. E., Stewart, M. C., Murray, G. D., . . Price, J. F. (2010). Association between polymorphisms of the dopamine receptor D2 and catechol-O-methyl transferase genes and cognitive function. Behavior Genetics, 40, 630-638. doi: 10.1007/s10519-010-9372-y

Brehmer, Y., Westerberg, H., Bellander, M., Fürth, D., Karlsson, S., \& Bäckman, L. (2009). Working memory plasticity modulated by dopamine transporter genotype. Neuroscience Letters, 467, 117-120. doi: 10.1016/j.neulet.2009.10.018

Colzato, L. S., van den Wildenberg, W. P. M., \& Hommel, B. (2013). Cognitive control and the COMT Val(158)Met polymorphism: Genetic modulation of videogame training and transfer to task-switching efficiency. Psychological Research. doi:10.1007/s00426-013-0514-8

Colzato, L. S., Waszak, F., Nieuwenhuis, S., Posthuma, D., \& Hommel, B. (2010). The flexible mind is associated with the catechol-Omethyltransferase (COMT) Val 158 Met polymorphism: Evidence for a role of dopamine in the control of task-switching. Neuropsychologia, 48, 2764-2768. doi:10.1016/j.neuropsychologia.2010.04.023

Cropley, V. L., Fujita, M., Innis, R. B., \& Nathan, P. J. (2006). Molecular imaging of the dopaminergic system and its association with human cognitive function. Biological Psychiatry, 59(10), 898-907. doi: 10.1016/j.biopsych.2006.03.004

Dahlin, E., Stigsdotter Neely, A., Larsson, A., Bäckman, L., \& Nyberg, L. (2008). Transfer of learning after updating training mediated by the striatum. Science, 320, 1510-1512. doi:10.1126/science.1155466

Deary, I. J., Johnson, W., \& Houlihan, L. M. (2009). Genetic foundations of human intelligence. Human Genetics, 126, 215-232. doi:10.1007/ s00439-009-0655-4

Friedman, N., Miyake, A., Young, S., DeFries, J., Corley, R., \& Hewitt, J. (2008). Individual differences in executive functions are almost entirely genetic in origin. Journal of Experimental Psychology: General, 137, 201-225. doi:10.1037/0096-3445.137.2.201

Friling, S., Andersson, E., Thompson, L., Jonssonc, M., Hebsgaard, J., Nanou, E., ... Ericson, J. (2009). Efficient production of mesencephalic dopamine neurons by Lmx1a expression in embryonic stem cells. PNAS Proceedings of the National Academy of Sciences of the United States of America, 106, 7613-7618. doi:10.1073/pnas.0902396106

Grace, A. A. (1991). Phasic versus tonic dopamine release and the modulation of dopamine system responsivity: A hypothesis for the etiology of schizophrenia. Neuroscience, 41, 1-24. doi:10.1016/03064522(91)90196-U

Hirvonen, M., Laakso, A., Någren, K., Rinne, J. O., Pohjalainen, T., \& Hietala, J. (2004). C957T polymorphism of the dopamine D2 receptor (DRD2) gene affects striatal DRD2 availability in vivo. Molecular Psychiatry, 9, 1060-1061. doi:10.1038/sj.mp.4001561

Hirvonen, M., Laakso, A., Någren, K., Rinne, J. O., Pohjalainen, T., \& Hietala, J. (2005). C957T polymorphism of the dopamine D2 receptor (DRD2) gene affects striatal DRD2 availability in vivo. Molecular Psychiatry, 9, 1060-1061. doi:10.1038/sj.mp.4001561

Hirvonen, M., Lumme, V., Hirvonen, J., Pesonen, U., Någren, K., Vahlberg, T., . . Hietala, J. (2009). C957T polymorphism of the human dopamine D2 receptor gene predicts extrastriatal dopamine receptor availability in vivo. Progress in Neuro-Psychopharmacology \& Biological Psychiatry, 33, 630-636. doi:10.1016/j.pnpbp.2009.02.021
Hu, L., \& Bentler, P. (1999). Cutoff criteria for fit indexes in covariance structure analysis: Conventional criteria versus new alternatives. Structural Equation Modeling: A Multidisciplinary Journal, 6, 1-55. doi: 10.1080/10705519909540118

Jäger, A. O., Süß, H.-M., \& Beauducel, A. (1997). Berliner Intelligenzstruktur-Test, BIS-Test. Form 4. Handanweisung. Göttingen, Germany: Hogrefe.

Jolles, D. D., Grol, M. J., Van Buchem, M. A., Rombouts, S. A. R. B., \& Crone, E. A. (2010). Practice effects in the brain: Changes in cerebral activation after working memory practice depend on task demands. NeuroImage, 52, 658-668. doi:10.1016/j.neuroimage.2010.04.028

Krugel, L. K., Biele, G., Mohr, P. N. C., Li, S.-C., \& Heekeren, H. R. (2009). Genetic variation in dopaminergic neuromodulation influences the ability to rapidly and flexibly adapt decisions. PNAS Proceedings of the National Academy of Sciences of the United States of America, 106, 17951-17956. doi:10.1073/pnas.0905191106

Li, S.-C., Papenberg, G., Nagel, I. E., Preuschhof, C., Schröder, J., Nietfeld, W., . . . Bäckman, L. (2013). Aging magnifies the effects of dopamine transporter and D2 receptor genes on backward serial memory. Neurobiology of Aging, 34, 358.e1-358.e10. doi:10.1016/j .neurobiolaging.2012.08.001

Lindenberger, U., Nagel, I. E., Chicherio, C., Li, S.-C., Heekeren, H. R., \& Bäckman, L. (2008). Age-related decline in brain resources modulates genetic effects on cognitive functioning. Frontiers in Neuroscience, 2. doi:10.3389/neuro.01.039.2008

Lövdén, M., Bäckman, L., Lindenberger, U., Schaefer, S., \& Schmiedek, F. (2010). A theoretical framework for the study of adult cognitive plasticity. Psychological Bulletin, 136, 659-676. doi:10.1037/a0020080

McArdle, J. J., \& Nesselroade, J. R. (1994). Using multivariate data to structure developmental change. In S. H. Cohen \& H. W. Reese (Eds.), Life-span developmental psychology: Methodological contributions (pp. 223-267). Hillsdale, NJ: Erlbaum.

Mercado, E. (2008). Neural and cognitive plasticity: From maps to minds. Psychological Bulletin, 134, 109-137. doi:10.1037/0033-2909.134.1 .109

Meredith, W., \& Teresi, J. A. (2006). An essay on measurement and factorial invariance. Medical Care, 44, S69-77. doi:10.1097/01.mlr .0000245438 .73837 .89

Muthén, L. K., \& Muthén, B. O. (1998-2012). Mplus user's guide (7th ed.). Los Angeles, CA: Muthén \& Muthén.

Nolan, K. A., Bilder, R. M., Lachman, H. M., \& Volavka, J. (2004). Catechol O-methyltransferase Val(158)Met polylmorphism in schizophrenia: Differential effects of Val and Met alleles on cognitive stability and flexibility. The American Journal of Psychiatry, 161, 359-361. doi:10.1176/appi.ajp.161.2.359

Papassotiropoulos, A., \& de Quervain, D. J.-F. (2011). Genetics of human episodic memory: Dealing with complexity. Trends in Cognitive Sciences, 15, 381-387. doi:10.1016/j.tics.2011.07.005

Papenberg, G., Bäckman, L., Nagel, I. E., Nietfeld, W., Schröder, J., Bertram, L., . . Li, S.-C. (2013). Dopaminergic gene polymorphisms affect long-term forgetting in old age: Further support for the magnification hypothesis. Journal of Cognitive Neuroscience, 25, 571-579. doi:10.1162/jocn_a_00359

Posthuma, D., Mulder, E. J. C. M., Boomsma, D. I., \& de Geus, E. J. C. (2002). Genetic analysis of IQ, processing speed and stimulus-response incongruency effects. Biological Psychology, 61, 157-182. doi:10.1016/ S0301-0511(02)00057-1

Savitz, J., Solms, M., \& Ramesar, R. (2006). The molecular genetics of cognition: Dopamine, COMT and BDNF. Genes, Brain, \& Behavior, 5 , 311-328. doi:10.1111/j.1601-183X.2005.00163.X

Schjeide, B.-M. M., Schnack, C., Lambert, J.-C., Lill, C. M., Kirchheiner, J., Tumani, H., . . Bertram, L. (2011). The role of clusterin, complement receptor 1, and phosphatidylinositol binding clathrin assembly protein in Alzheimer disease risk and cerebrospinal fluid biomarker levels. Ar- 
chives of General Psychiatry, 68, 207-213. doi:10.1001/archgenpsychiatry.2010.196

Schmiedek, F., Lövdén, M., \& Lindenberger, U. (2010). Hundred days of cognitive training enhance broad cognitive abilities in adulthood: Findings from the COGITO study. Frontiers in Aging Neuroscience, 2. doi:10.3389/fnagi.2010.00027

Sheynikhovich, D., Otani, S., \& Arleo, A. (2011). The role of tonic and phasic dopamine for long-term synaptic plasticity in the prefrontal cortex: A computational model. Journal of Physiology - Paris, 105, 45-52. doi:10.1016/j.jphysparis.2011.08.001

Stroth, S., Reinhardt, R. K., Thöne, J., Hille, K., Schneider, M., Härtel, S., . . Spitzer, M. (2010). Neurobiology of Learning and Memory Impact of aerobic exercise training on cognitive functions and affect associated to the COMT polymorphism in young adults. Neurobiology of Learning and Memory, 94, 364-372. doi:10.1016/j.nlm.2010.08.003

Tunbridge, E. M., Harrison, P. J., \& Weinberger, D. R. (2006). CatecholO-methyltransferase, cognition, and psychosis: Val 158 met and beyond. Biological Psychiatry, 60, 141-151. doi:10.1016/j.biopsych.2005.10 .024

Wright, M., De Geus, E., Ando, J., Luciano, M., Posthuma, D., Ono, Y., . . Boomsma, D. (2001). Genetics of cognition: Outline of a collaborative twin study. Twin Research, 4, 48-56. doi:10.1375/1369052012146

Received January 28, 2014

Revision received March 25, 2014

Accepted March 26, 2014 